\title{
Feasibility of a Randomized Controlled Trial of Self-Administered Acupressure for Symptom Management in Older Adults with Knee Osteoarthritis
}

\author{
Lydia W. Li, PhD, ${ }^{1}$ Richard E. Harris, PhD, ${ }^{2}$ Susan L. Murphy, ScD, OTR, \\ Alex Tsodikov, $\mathrm{PhD}^{4}$, and Laura Struble, $\mathrm{PhD}^{5}$
}

\begin{abstract}
Objectives: To assess the feasibility of a study to evaluate the efficacy of self-administered acupressure in pain and related symptom management for older people with symptomatic knee osteoarthritis. Feasibility with regard to (1) sample recruitment and retention, (2) treatment fidelity and adherence, and (3) tolerability and adverse events was examined.

Methods: The study was a randomized controlled trial. Community-living older adults were recruited and randomly assigned to one of three groups: verum acupressure, sham acupressure, and usual care. Participants in the first two groups learned their respective acupressure protocol during their first center visit and from a set of materials. They were asked to practice the protocol at home once daily, 5 days a week, for 8 weeks. Participants attended three center visits and received weekly phone calls from a research assistant in an 8-week study period. Both quantitative and qualitative data collected from center visits and weekly phone calls were used to examine study feasibility.
\end{abstract}

Results: A total of 150 participants (mean age, 73 years; $38 \%$ men) were enrolled; $83 \%$ completed all three center visits. Among those assigned to verum and sham acupressure groups, $94 \%$ passed a fidelity check at the second visit, more than $80 \%$ reported performing self-administered acupressure as instructed most of the time, and about $10 \%$ reported discomfort from performing the acupressure. Thirty adverse events were reported; most were related to pre-existing health conditions.

Conclusions: It is feasible to conduct a study of self-administered acupressure for symptom management in community-living older adults with knee osteoarthritis, although sample recruitment may be challenging.

\section{Introduction}

$\mathbf{K}$ NEE OSTEOARTHRITIS (OA) IS RAPIDLY increasing in frequency, in part because the population is becoming older and more obese. ${ }^{1}$ The effect of persistent knee pain on quality of life is substantial. ${ }^{2}$ Prior studies have shown that at least half of older adults with knee pain report some restriction of daily activities and often present with other comorbid symptoms, including fatigue, sleep, and mood disturbance. ${ }^{3}$ OA is also responsible for a substantial economic burden. In 2005 , the cost of hospitalization for musculoskeletal procedures was estimated at $\$ 31.5$ billion, totaling $10 \%$ of overall inpatient costs. ${ }^{4}$ Without advances in prevention and treat- ment, the magnitude of this problem will continue to grow, as the number of total knee joint replacements is anticipated to rise $673 \%$ by $2030 .^{5}$

Although many options are available to persons with knee OA (e.g., medications, joint injections, physical therapy), current data suggest that their therapeutic effect is less than desirable. ${ }^{6}$ In meta-analyses of randomized controlled trials (RCTs) examining pharmacologic treatments in OA, acetaminophen leads to an average of $4-\mathrm{mm}$ improvement compared with placebo (0-100-mm scale), whereas trials of nonsteroidal anti-inflammatory drugs that do not exclude nonresponders show an average of $8-\mathrm{mm}$ improvement compared with placebo. ${ }^{7,8}$ These effects and effect sizes are

${ }^{1}$ School of Social Work, University of Michigan, Ann Arbor, MI.

${ }^{2}$ Department of Anesthesiology, University of Michigan, Ann Arbor, MI.

${ }^{3}$ Department of Physical Medicine and Rehabilitation, University of Michigan, Ann Arbor, MI.

${ }^{4}$ Department of Biostatistics, School of Public Health, University of Michigan, Ann Arbor, MI.

${ }^{5}$ School of Nursing, University of Michigan, Ann Arbor, MI. 
small. In addition, an array of adverse effects, such as cardiovascular or renal complications, are associated with longterm nonsteroidal anti-inflammatory drug use. ${ }^{9}$ Many older adults have comorbid conditions that would dictate their drug therapy ${ }^{10}$ and polypharmacy leads to drug interactions. Research on nonpharmacologic interventions for pain has shown promising results, although some (e.g., physical therapy, cognitive-behavioral therapy) are costly because they require professional practitioners to administer the therapy. In aggregate, these facts suggest that effective, safe, and low-cost nonpharmacologic treatments for older adults with knee OA are greatly needed. Self-administered acupressure has the potential to be one such alternative treatment.

Acupressure is a Traditional Chinese Medicine (TCM) technique based on a philosophy similar to that of acupuncture. In contrast to acupuncture, which uses very fine needles, acupressure involves using fingers or other devices to apply pressure on different acupuncture points (acupoints) to stimulate meridians and increase the flow of $q i$ (life energy). Acupressure is safer and less invasive; in addition, once patients learn how to administer the protocol, they require little or no assistance to complete treatment. In contrast, a trained TCM specialist must administer acupuncture.

Systematic reviews of acupressure for symptom management concluded that although most studies showed positive effects of acupressure on their primary outcomes, their methodological quality was moderate to low ${ }^{11-14}$ Most studies involved practitioner-administered acupressure. Among those that focused on self-administered acupressure, older adults were underrepresented. ${ }^{15}$ Recently, Zhang et al. reported a pilot study of training postmenopausal women with knee OA pain to self-administer acupressure. ${ }^{16}$ Their sample consisted of mostly young-old women (mean age, 61 years), was small ( $n=36)$, and had high dropout rates $(33 \%)$. They did not report fidelity or adherence, and few previous self-administered acupressure studies have addressed these issues.

The current RCT was conducted to examine the efficacy and feasibility of self-administered acupressure for symptom management in people age 65 years and older with symptomatic knee OA. This report focuses on evaluating the study feasibility of (1) sample recruitment and retention, (2) treatment fidelity and adherence, and (3) tolerability and adverse events.

\section{Materials and Methods}

\section{Design and intervention}

The RCT involved three groups: verum acupressure, sham acupressure, and usual care (UC). The intervention lasted 8 weeks. Participants attended three visits - the first fourth, and eighth week - at our center. Participants also received weekly phone calls from a research assistant (RA) during the 8 weeks. The investigators' university human subjects review board approved the study, and all participants provided informed consent.

\section{Training of acupressure protocol}

RAs were trained to perform both verum and sham acupressure protocols by a co-investigator (R.E.H.) who was certified in acupuncture by the National Certification Com- mission for Acupuncture and Oriental Medicine. They had to pass a trial evaluation from this investigator before having any participant contact. All RAs were blinded to the assignment of verum and sham acupressure. Each participant was trained individually by an RA during their first center visit and was asked to self-apply the acupressure protocol at home once daily, 5 days per week, for 8 weeks. The instruction continued until the participant demonstrated greater than $90 \%$ accuracy on a competency checklist that included 12 items (9 items about location of the acupoints, 1 about proper pressure and stimulation, 1 about stimulation duration, and 1 about frequency of acupressure sessions.) Greater than $90 \%$ accuracy meant being correct in at least 11 of the 12 items.

To help the participants learn the self-administered acupressure protocol at home, the following materials were given: (1) a wooden hand-held device designed for acupressure (Acu-Ki, Bodytools, Middletown, CA), which we thought would make it easier for participants to apply pressure; (b) a DVD that shows an older person performing the assigned acupressure protocol with instruction and explanation from R.E.H.; (3) a diagram of the assigned acupoints with written instructions on how to locate them; and (4) a timer to track time upon self-administering the acupressure protocol. Acupressure group participants received these materials at the end of their first visit.

All participants received weekly telephone calls from an RA, who addressed questions related to study participation and asked about adverse events, tolerability with acupressure, and changes in medications and treatment regimen. Retraining was offered if participants expressed difficulty mastering the acupressure protocol.

\section{Verum acupressure group}

Participants assigned to verum acupressure were taught to apply pressure, using the hand-held device, to nine acupoints on their body (Fig. 1): Yintang, Anmian, HT 7, SP 6, and KI 3. HT 7, SP 6, and KI 3 were stimulated bilaterally. Each point should receive 3 minutes of continuous pressure in clockwise or counterclockwise circles. The pressure should be sufficient to evoke a de qi sensation (i.e., dull ache, tingling, soreness). The acupressure protocol was developed by R.E.H. in consultation with other local acupuncturists. The points were chosen for their ability to reduce fatigue and sleep disturbance, common comorbidities in patients with OA and pain. ${ }^{3}$ This approach was relatively novel in that the hope was to indirectly improve pain and other symptoms by targeting fatigue and sleep symptoms.

\section{Sham acupressure group}

Participants assigned to sham acupressure received similar instruction as those assigned to verum acupressure, except that they were taught to apply pressure to 9 points that were not on acupuncture meridians or known acupuncture points (Fig. 2).

\section{UC group}

UC participants did not receive acupressure but had weekly check-in phone calls. They had an opportunity to learn both verum and sham acupressure protocols at the end of study participation. Both types of acupressure were 
FIG. 1. Point locations for verum acupressure.

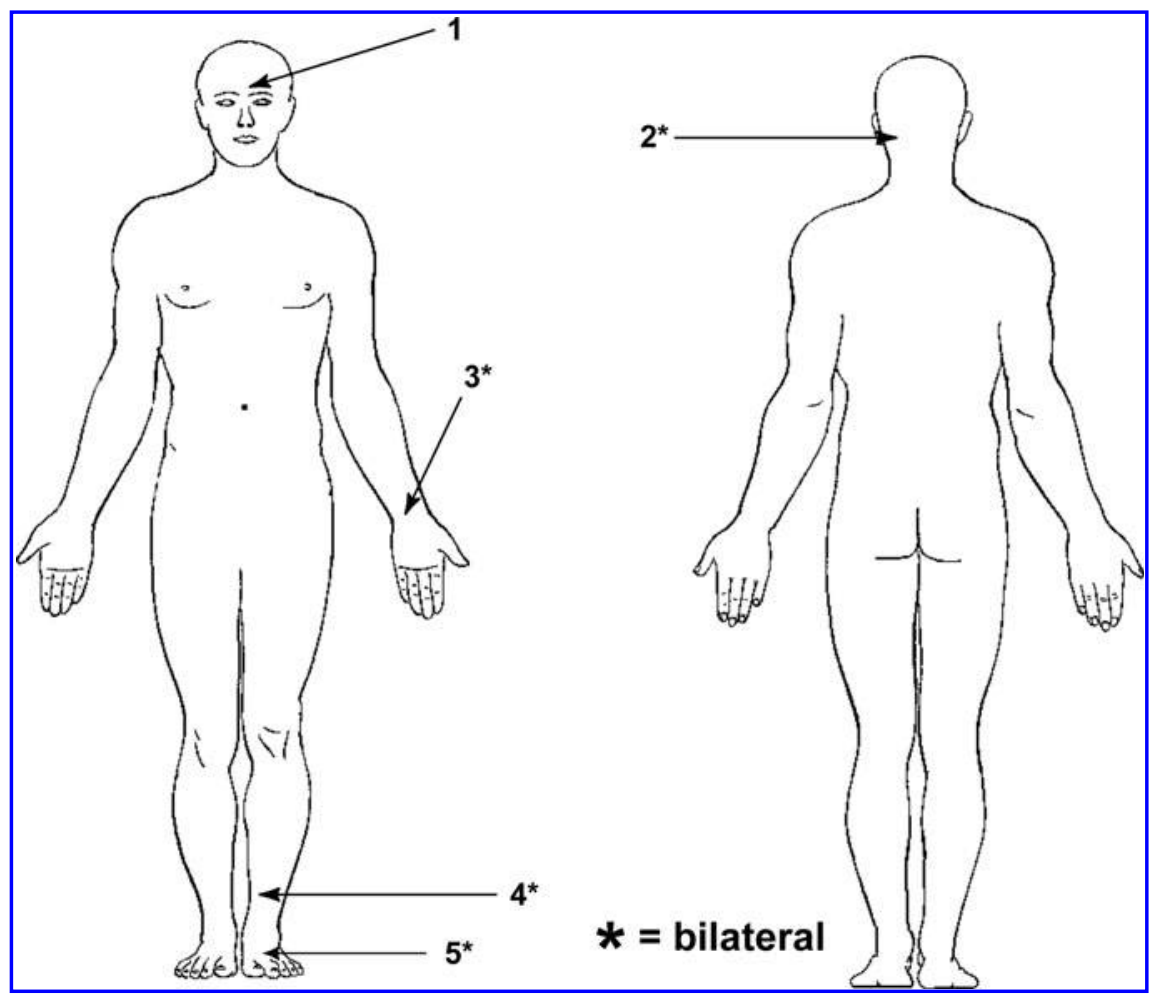

offered so that RAs, as well as participants, remained blinded to acupressure group assignment.

\section{Sample}

The target population was community-living older adults with symptomatic knee OA. Table 1 lists the inclusion and exclusion criteria. The exclusion criteria were set to reduce confounding effects and ensure participant safety.

Randomization, blinding, and allocation concealment

On the basis of a power analysis, a sample size of 180 was planned, with 60 patients in each group, using block

FIG. 2. Point locations for sham acupressure.

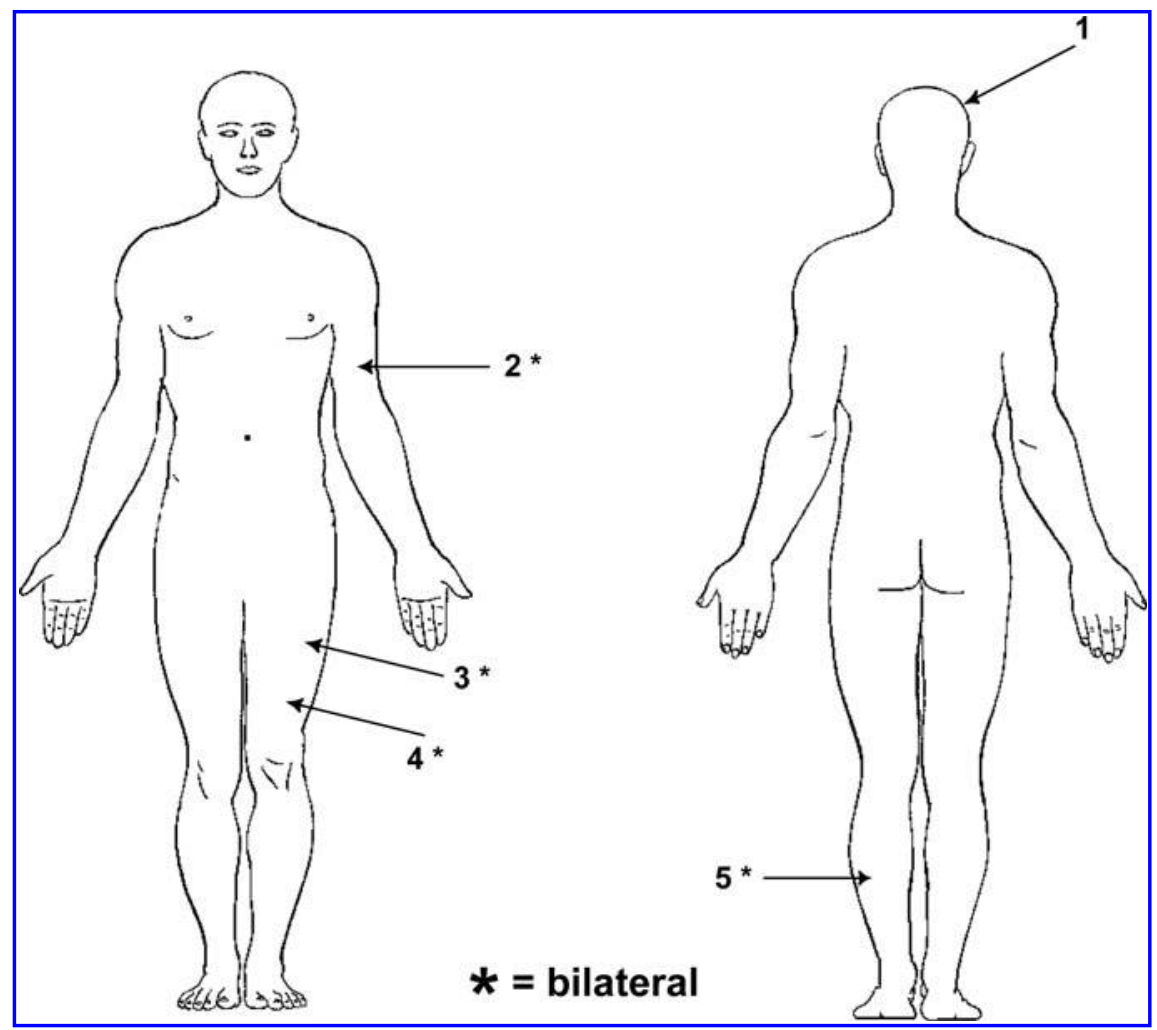


Table 1. Participant Inclusion and Exclusion CRiteria

Inclusion criteria Exclusion criteria

Age 65 yr or older

Community-living (i.e., own home, senior residence, apartment)

Meets the American College of Rheumatology clinical criteria for knee osteoarthritis ${ }^{17}$

Have moderate to severe knee pain (pain $\geq 3$ on $0-10$ Visual Analogue Scale) that has lasted for 3 mo or longer Body-mass index $\leq 45$

Ability to speak and write in English

Adequate cognitive status (score $>5$ on the 6 -item screener) ${ }^{23}$

Adequate functional ability to administer the acupressure protocol (e.g., able to use fingers or device to apply pressure to acupoints, able to easily reach feet to access acupoints)

Ability to understand the treatment protocol through demonstration after being instructed

Ambulatory with or without an assistive device

Adequate hearing and vision to follow study protocol

Have a telephone and television

randomization in blocks of 3. A randomization list was computer-generated by the study biostatistician. All research personnel were blinded to the randomization list.

\section{Recruitment}

Several methods were used to recruit participants, including advertising in local newspapers and magazines; posting flyers at health clinics, senior centers, grocery stores, and restaurants; asking referrals from rheumatologists; setting up an alert system at physician visits with a university-affiliated geriatric clinic; and sending letters to previous research participants with knee OA. To compensate time and travel costs, checks of $\$ 25, \$ 15$, and $\$ 25$, respectively, were sent to participants after their first, second, and third visits.

\section{Screening and enrollment}

Initial screening was done over the telephone. Those deemed eligible were invited to our center for a second-level eligibility assessment conducted by an RA who had been trained to use the American College of Rheumatology (ACR) clinical criteria for knee osteoarthritis to assess eligibility. ${ }^{17}$

\section{Data collection}

Center visits. At the first of three visits, participants completed questionnaires that included demographic ques-
Have cancer or received cancer treatment within last 6 mo (exception: skin cancer where the location is not around acupoints)

Have any bleeding diathesis conditions or taking anticoagulant/antiplatelet medications

Have health conditions that could confound the effect of acupressure (e.g., rheumatoid arthritis, lupus, multiple sclerosis, diabetic neuropathy, peripheral neuropathy, Parkinson disease, limb paralysis)

Have ever had knee replacement surgery

Have received the following treatments or therapies in the last 2 mo: occupational or physical therapy, acupuncture or acupressure therapy, opioid therapy, cognitive-behavior therapy, arthritis self-management programs, arthroscopic procedure, knee injection

Planned or scheduled new treatment for knee pain in the next $3 \mathrm{mo}$

Regular current use of narcotics or centrally acting agents (including Pristiq, Duragesic, Fentora, Actiq, hydrocodone, Lorcet/Lortab, Norco, codeine, hydromorphone, Dilaudid, Demerol, Exalgo, methadone, tramadol, Ultram, meperidine, Demerol, Dolophine, Methadose, Percocet, morphine, MS Contin, oxycodone, Oxycontin, fentanyl) tions and outcome measures, including pain (e.g., Western Ontario and McMaster University Arthritis Index ${ }^{18}$ ), physical function, quality of life, fatigue, and self-efficacy. In addition, performance-based measures of physical function (e.g., Timed Up and Go test ${ }^{19}$ ) were administered by an RA. The second visit was scheduled about 4 weeks after to check treatment fidelity. Participants in acupressure groups demonstrated to an RA their assigned acupressure protocol. The final visit was 8 weeks after the first. Outcome measures were assessed at both the second and final visits.

Weekly phone calls. During the weekly telephone calls, an RA asked participants to rate their pain in the previous week on a $0-10$ rating scale. Acupressure groups participants were asked about any physical discomfort from performing self-administered acupressure and to rate the extent to which they adhered to self-practice. The RA kept a log of each phone contact.

\section{Data analysis}

Data from all center visits and telephone calls were used to evaluate feasibility. Descriptive statistics, $t$ tests, and analysis of variance were conducted by using Stata software, version 13 (Stata Corp., College Station, TX). ${ }^{20}$ Qualitative data were coded and categorized. 


\section{Results}

\section{Sample recruitment and retention}

Figure 3 presents the study participant flow. Over 18 months, 412 people underwent telephone screening. More than half $(n=211)$ did not meet eligibility criteria. The primary reasons included receiving or recently received other treatments (e.g., physical therapy, knee injection), having confounding health conditions (e.g., rheumatoid arthritis, multiple sclerosis), taking anticoagulant/antiplatelet medications, previous knee replacement, and age younger than 65 years. Among the 201 people referred to level 2 screening, 19 were excluded for similar reasons. In total, 150 adults were enrolled, with 50 assigned to each group.

Sample characteristics are shown in Table 2. The participants ranged in age from 65 to 96 years (mean, 73 years), and $38 \%$ were men. Eighty-eight percent were white, $82 \%$ had college or higher degrees, and $67 \%$ had annual income of USD 50,000 or higher. There were no significant between group differences in almost all sociodemographic characteristics, except that the verum group had more nonwhites than did the UC group. The number of nonwhites in the sample was small $(n=10,8$, and 2 for verum, sham, and UC groups, respectively).

The retention rate was high- $-86 \%$ at visit 2 and $83 \%$ at visit 3 (Fig. 3). Attrition rates did not differ among the three groups. Major reasons for withdrawal included interference of other health conditions, seeking other therapies for pain, view that acupressure was too time-consuming, and loss of interest. Socioeconomic status or baseline measures of pain did not significantly differ between those who dropped out and those who completed the study, except that a higher percentage of nonwhites $(33 \%)$ than whites $(14 \%)$ withdrew.

\section{Treatment fidelity and adherence}

The time needed for acupressure training during the first center visit was about 30 minutes. For most participants, this training plus the sent-home materials (DVD and printed diagram) seemed adequate. Participants also used the weekly phone calls to clarify any confusion about their assigned acupressure protocol. Two participants returned to the center for retraining.
FIG. 3. Flow of participants through the study.

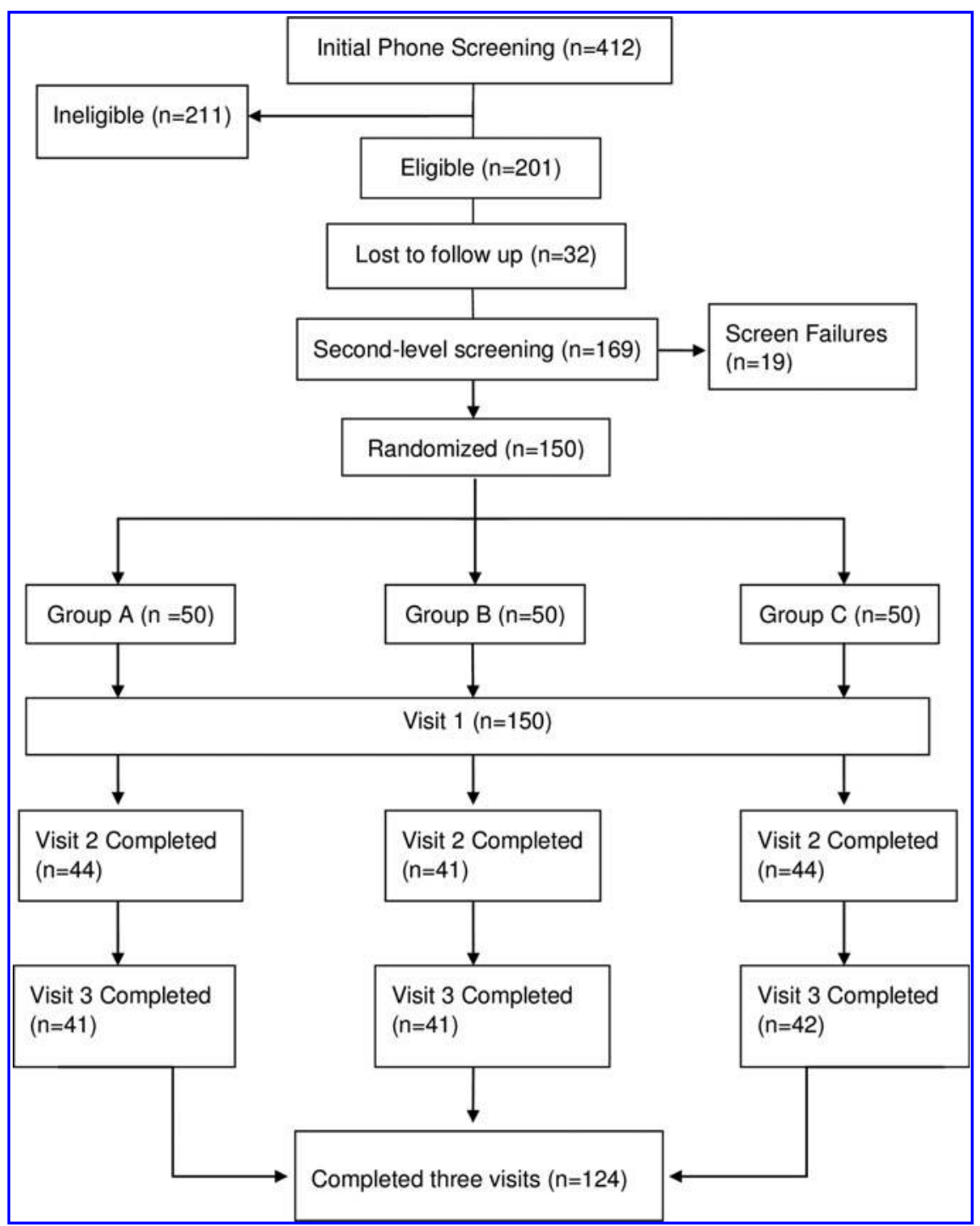


Table 2. Participants Characteristics

\begin{tabular}{|c|c|c|}
\hline Characteristic & Value & Valid $\mathrm{n}$ \\
\hline Age (range, 65-96 yr) & $72.7 \pm 6.5 \mathrm{yr}$ & 150 \\
\hline Women & $62.2 \%$ & 148 \\
\hline Race & & 146 \\
\hline White & $88.4 \%$ & \\
\hline Black & $7.5 \%$ & \\
\hline Asian & $2.7 \%$ & \\
\hline Other & $1.4 \%$ & \\
\hline Education & & 148 \\
\hline Graduate degree & $49.3 \%$ & \\
\hline Associate/bachelor degree & $33.1 \%$ & \\
\hline Some college, no degree & $11.5 \%$ & \\
\hline High school/ GED & $6.1 \%$ & \\
\hline Income & & 131 \\
\hline$\leq \$ 29,999$ & $13.7 \%$ & \\
\hline$\$ 30,000-\$ 49,999$ & $19.1 \%$ & \\
\hline$\$ 50,000-\$ 69,999$ & $22.9 \%$ & \\
\hline$\$ 70,000-\$ 89,999$ & $16.0 \%$ & \\
\hline$\geq \$ 90,000$ & $28.3 \%$ & \\
\hline Employment & & 147 \\
\hline Retired & $80.2 \%$ & \\
\hline Part-time/seasonal & $10.9 \%$ & \\
\hline Full-time & $8.2 \%$ & \\
\hline Never gainfully employed & $0.7 \%$ & \\
\hline Marital status & & 146 \\
\hline Married & 54.8 & \\
\hline Divorced/separated & 23.3 & \\
\hline Widowed & 11.7 & \\
\hline Never married & 7.5 & \\
\hline Other & 2.7 & \\
\hline $\begin{array}{l}\text { Body-mass index } \\
\quad\left(\text { range, } 18.1-45.7 \mathrm{~kg} / \mathrm{m}^{2}\right)\end{array}$ & $29.1 \pm 5.6 \mathrm{~kg} / \mathrm{m}^{2}$ & 150 \\
\hline
\end{tabular}

Values expressed with a plus/minus sign are the mean \pm standard deviation.

During the fidelity check at the second visit, $96 \%$ of participants achieved $100 \%$ accuracy on the competency checklist. Only 5 out of 129 missed the location of a couple of acupoints. The DVD and printed diagram were said to be useful in helping to locate the points and practice at home.
Two self-report measures were used to assess participants' treatment adherence. The first was via phone calls. Participants were asked how often they were able to perform the acupressure at home (all of the time, some of the time, or none of the time). Figure 4 shows that across all phone contacts, between $81 \%$ and $90 \%$ of respondents said "most of the time."

Adherence was also assessed during the second and third center visits by using a four-item scale adapted from the Medical Outcomes Study. ${ }^{21}$ Each item was rated from 1 (none of the time) to 6 (all of the time). Two items were reverse-coded. A sample item is: "I followed the instruction exactly to practice acupressure at home." The mean scale scores were 5.3 and 5.5 at visits 2 and 3 , respectively, suggesting high adherence. This finding is consistent with results from the phone-administered measure. The two acupressure groups had no significant differences in either measure of adherence.

\section{Tolerability and adverse events}

To assess tolerability, participants were asked during weekly phone calls whether they experienced discomfort from performing acupressure. Figure 5 shows that at the first call, $10.5 \%$ reported experiencing discomfort. The percentage decreased over time except at the sixth call, when 3 out of $72(4.2 \%)$ gave affirmative responses.

Participant-reported discomfort can be classified into major and rare types. The major ones were soreness/tenderness/achiness, pain, and bruising. The rare ones, which one to three people reported, included tingling, blistering, muscle spasm, and broken skin. Most people who reported discomfort felt the problem was mild, and most problems disappeared after the participant followed the RA's advice to modify the way pressure was applied. Some participants had used the hand-held device incorrectly. The device had a pointy and a flat end, and they were supposed to use the flat end. Some used the pointy end, which caused broken skin.

Thirty adverse events were noted; three were judged to be related to the study and one possibly related to the study. All three related adverse events were broken skin and soreness in acupoint areas, possibly caused by incorrect use of the

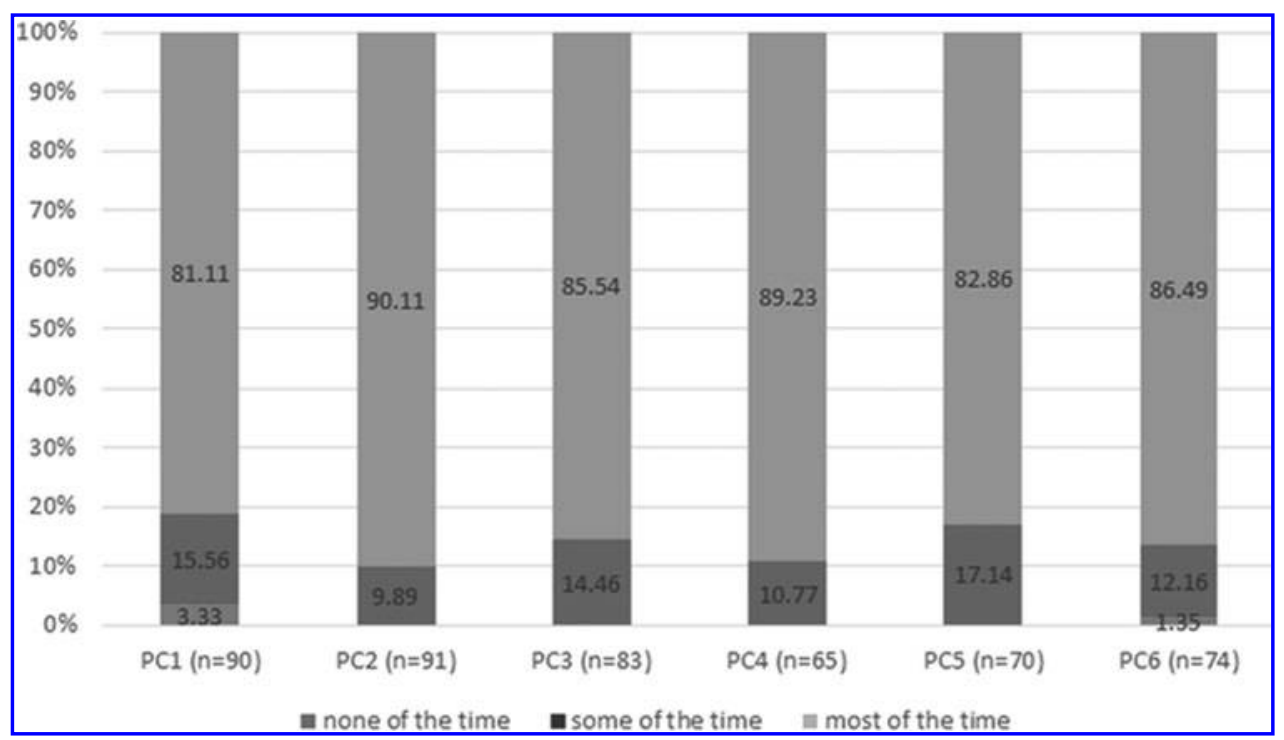

FIG. 4. Distribution of response to the adherence question during phone calls. Only participants in the two acupressure groups were asked to rate their adherence to perform the assigned protocol at home. PC $x$ refers to the $x$ (first, second...) phone call. The number of participants $(n)$ responding to the adherence question in each phone contact varied. 


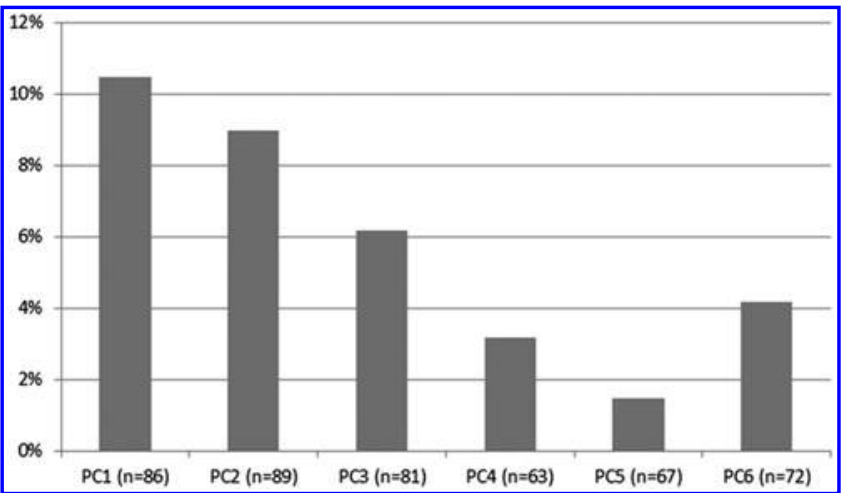

FIG. 5. Percentage of participants who, during phone call follow-up, reported discomfort from performing acupressure. Only participants in the two acupressure groups were asked whether they were having discomfort from performing acupressure. The number of participants $(n)$ responding to the question in each phone contact varied.

hand-held device. The possibly related adverse event was thigh pain, which led to an emergency department visit. The cause of pain was unknown; therefore, it was classified as possibly related.

The adverse events classified as unrelated to the study included a variety of medical occurrences: fall, urinary tract infection, sinus infection, and others. No pattern in these events was identified.

\section{Discussion}

Little is known about using self-administered acupressure as an alternative therapy in older adults. This RCT to evaluate the feasibility and efficacy of using self-administered acupressure to manage symptoms for older people with knee OA has highlighted the potential and challenges for future work.

\section{Challenges of sample recruitment}

One hundred and fifty older adults were recruited, 17\% below our target. The process of sample recruitment was challenging, in part because of weather constraints. In winter months, many older adults in the area relocated themselves to warmer places. About $83 \%$ of participants were enrolled between May and October. Future studies should consider the influence of weather on older people's motivation to participate.

Among the methods used to publicize the study, the most effective one was advertising in local newspapers and magazines, probably because older people still prefer these media for news and information. However, advertisement can reach a saturation point - the number of calls to the tollfree number decreased over time despite consistent advertisement. Thus, a variety of methods is needed. Advertisement is costly. A realistic amount for such an expenditure should be included in the budget. Finally, dedicated personnel for recruitment are needed because the task is time and energy consuming.

There was high interest in acupressure, but many individuals were excluded because they did not meet eligibility criteria. Future studies may consider dropping some exclusion criteria, such as recent receipt of occupational/physical therapy. Reducing the age threshold to 60 years would also increase the potential participant pool. The requirement of three in-person visits may have deterred some older adults from participation, as they may no longer drive. It may help if assessments are conducted in their homes. However, this may be difficult when performance-based assessments that require standardized equipment or measured walking courses are used.

Compared with previous studies of self-administered acupressure, ${ }^{15,16}$ the sample included an older population, particularly older men, that has not been studied before. However, the sample was biased toward whites and people with higher socioeconomic status. Future studies should broaden the sample to ethnic minorities and people with low socioeconomic status. These groups may most benefit from a low-cost and easy-to-administer alternative treatment for symptom management.

\section{Sample retention}

Of enrolled participants, $83 \%$ were retained. The low attrition rate, especially in comparison with Zhang and colleagues' study, ${ }^{16}$ was likely attributed to two factors. One was that the RA in the current study could address participants' questions during weekly phone calls, an especially important factor in the early weeks of participation. The calls may have lessened frustration and sustained interest. Second, compensation for costs of coming to the center may be important for those with low or fixed incomes. Among those who withdrew, interference of other health problems was a major reason. This is a challenge as the targeted population tends to have multiple health conditions.

\section{Training and adherence}

The training of self-administered acupressure was effective. Participants considered the DVD and printed diagram as very helpful. Additionally, weekly phone calls reinforced participants' learning and treatment adherence. However, calls are labor intensive.

\section{Adverse events}

This study shows that self-administered acupressure is a safe and tolerable intervention for older people, which corroborates other acupressure studies. ${ }^{16,22}$ The hand-held device caused some discomfort and adverse events. Future studies should consider using other tools or just fingers to apply pressure. Despite adverse events, most were not serious and were unrelated to the study. Adverse events may be inevitable because this population is at risk for medical occurrence. A study-specific reporting plan that focuses on reporting adverse events possibly or likely related to study participation would be helpful in these types of studies.

\section{Conclusion}

On the basis of assessment of sample recruitment and retention, fidelity and adherence, and tolerability and adverse events, this study suggests that it is feasible to investigate the effect of self-administered acupressure on symptom management in community-living older adults with knee OA. 


\section{Acknowledgment}

The authors thank Kathy Scott, Ryan Scott, and Andrew Clauw for their assistance with conducting center visits and phone calls and data management. They also thank Melissa Krug, Angela Lyden, and Dana Arielle for their assistance with sample recruitment and study set-up. The study was supported by a grant from the Arthritis Foundation.

\section{Author Disclosure Statement}

No competing financial interests exist.

\section{References}

1. Gabriel SE, Michaud K. Epidemiological studies in incidence, prevalence, mortality, and comorbidity of the rheumatic diseases. Arthritis Res Ther 2009;11:229-229.

2. Dawson J, Linsell L, Zondervan K, et al. Impact of persistent hip or knee pain on overall health status in elderly people: a longitudinal population study. Arthritis Care Res 2005;53:368-374.

3. Murphy SL, Lyden AK, Phillips K, et al. Subgroups of older adults with osteoarthritis based upon differing comorbid symptom presentations and potential underlying pain mechanisms. Arthritis Res Ther 2011;13:R135.

4. Merrill C, Elixhauser A. Hospital stays involving musculoskeletal procedures, 1997-2005. 2007. Online document at: www.hcup-us.ahrq.gov/reports/statbriefs/sb34.jsp, accessed August 15, 2015.

5. Kurtz S, Ong K, Lau E, et al. Projections of primary and revision hip and knee arthroplasty in the United States from 2005 to 2030. J Bone Joint Surg 2007;89:780-785.

6. Zhang W, Nuki G, Moskowitz R, et al. OARSI recommendations for the management of hip and knee osteoarthritis: part III: changes in evidence following systematic cumulative update of research published through January 2009. Osteoarthritis Cartilage 2010;18:476-499.

7. Towheed T, Maxwell L, Judd M, et al. Acetaminophen for osteoarthritis. Cochrane Database Syst Rev 2006(1); CD004257.

8. Bjordal JM, Ljunggren AE, Klovning A, et al. Non-steroidal anti-inflammatory drugs, including cyclo-oxygenase-2 inhibitors, in osteoarthritic knee pain: meta-analysis of randomised placebo controlled trials. BMJ 2004;329:1317.

9. Friedewald VE, Bennett JS, Christo JP, et al. AJC Editor's consensus: selective and nonselective nonsteroidal antiinflammatory drugs and cardiovascular risk. Am J Cardiol 2010;106:873-884.

10. Seed SM, Dunican KC, Lynch AM. Treatment options for osteoarthritis: considerations for older adults. Hosp Pract 2011;39:62-73.
11. Jiang HR, Ni S, Li JL, et al. Systematic review of randomized clinical trials of acupressure therapy for primary dysmenorrhea. Evid Based Complement Alternat Med 2013;2013:169692.

12. Lee EJ, Frazier SK. The efficacy of acupressure for symptom management: a systematic review. J Pain Symptom Manage 2011;42:589-603.

13. Robinson N, Lorenc A, Liao X. The evidence for Shiatsu: a systematic review of Shiatsu and acupressure. BMC Complement Altern Med 2011;11:88.

14. Yeung W-F, Chung K-F, Poon MM-K, et al. Acupressure, reflexology, and auricular acupressure for insomnia: a systematic review of randomized controlled trials. Sleep Med 2012;13:971-984.

15. Song HJ, Seo H-J, Lee H, et al. Effect of self-acupressure for symptom management: a systematic review. Complement Ther Med 2015;23:68-78.

16. Zhang Y, Shen CL, Peck K, et al. Training selfadministered acupressure exercise among postmenopausal women with osteoarthritic knee pain: a feasibility study and lessons learned. Evid Based Complement Alternat Med 2012;2012:570431.

17. Altman R, Asch E, Bloch D, et al. Development of criteria for the classification and reporting of osteoarthritis: classification of osteoarthritis of the knee. Arthritis Rheum 1986;29:1039-1049.

18. Bellamy N. WOMAC: a 20-year experimental review of a patient-centered self-reported health status questionnaire. J Rheumatol 2002;29:2473-2476.

19. Podsiadlo D, Richardson S. The timed "Up \& Go": a test of basic functional mobility for frail elderly persons. $\underline{\mathrm{J} \mathrm{Am}}$ Geriatr Soc 1991;39:142-148.

20. Stata 13. College Station, TX: Stata Corp.; 2013.

21. Hays RD. Measures of patient adherence: RAND Medical Outcomes Study. 1994. Online document at: www.rand .org/health/surveys_tools/mos/mos_adherence.html, accessed April 24, 2013.

22. Zick SM, Alrawi S, Merel G, et al. Relaxation acupressure reduces persistent cancer-related fatigue. Evid Based Complement Alternat Med 2011;2011:142913.

Address correspondence to: Lydia W. Li, PhD

School of Social Work

University of Michigan

School of Social Work Building 1080 S. University

Ann Arbor, MI 48109-1106

E-mail: 1ydiali@umich.edu 
This article has been cited by:

1. Zidan Gong, Winnie Yu, Thomas Wong, Yuanqi Guo. Acupuncture/Acupressure for Knee Osteoarthritis (OA) Relieving in the Elderly: A Review 323-334. [CrossRef] 